\title{
COMMENT Growing health concerns from perfluoroalkyl substances: public health perspectives and clinical implications
}

\author{
Laura Anderko ${ }^{1}$ \\ Pediatric Research (2020) 87:980-981; https://doi.org/10.1038/s41390-020-0836-3
}

Developing fetuses can be exposed to perfluoroalkyl substances (PFAS) when maternal blood crosses the placenta and reaches the umbilical cord blood. Maternal serum PFAS concentrations during pregnancy are strongly correlated with cord serum concentrations. ${ }^{1}$ The longitudinal pregnancy cohort study conducted by Wikstrom et al. provides critical evidence in the effort to better understand the association between maternal levels of PFAS and birth outcomes. Increased prenatal maternal blood levels of PFAS (specifically, PFOS, PFOA, PFNA, PFDA and PFUnDA) were associated with lower birth weight, lower birth weight for gestational age, and being born small for gestational age. These associations were more pronounced and statistically significant in girls. These findings are powerful, especially considering a recent US study detected PFAS in the serum of all children in a nationally representative sample of 3- to 11 -year-olds. ${ }^{2}$

Most people in the United States and in other industrialized countries have measurable amounts of PFAS in their blood. PFOA and PFOS are the most prominent. The widespread nature of PFAS exposures from everyday consumer products and drinking water, coupled with the potential for long-term health impacts from these birth outcomes such as low birth weight, should elevate concerns worldwide. Evidence has shown that compared to normal weight infants, low-birth-weight infants may be at more risk for many future health problems such as intellectual and neurodevelopmental disabilities, obesity, and cardiovascular disease $^{3-7}$ The potential for widespread exposure and health impacts commands public health approaches to protect the health of children in the long term.

There is a critical need to reduce and eliminate exposures to improve the health of children over a lifetime. Since 1999, the National Health and Nutrition Examination Survey (NHANES) has measured blood PFAS in the U.S. population. Based on data collected from previous NHANES 3 cycle years, levels of PFOA and PFOS are generally decreasing in the blood of the general population. This appears to be a result of the 2006 agreement by eight major companies in the PFAS industry to phase outvoluntarily-these two PFAS by 2015.

However, drinking water, an important source of PFAS, is currently not regulated in the US. The US Environmental Protection Agency (EPA) has not yet issued an enforceable standard called a Maximum Contaminant Level (MCL). In 2016 the EPA established a lifetime Health Advisory Level (HAL), which is set at 70 parts per trillion (ppt) for the combined concentrations of PFOS and PFOA. HALs are not legally enforceable and are not designed to be a definitive health-effect level but rather provide a margin of protection over a lifetime. ${ }^{8,9}$ In the face of uncertainty and lack of federal regulation, some states are beginning to establish their own guidelines. For example, New York became the first state to adopt an MCL of 10 parts per trillion (ppt) for PFOA and $10 \mathrm{ppt}$ for PFOS, and in Minnesota a new PFOS value of $15 \mathrm{ppt}$ was established, which is well below the 70 ppt standard set by the EPA. ${ }^{10,11}$ The Association of State Drinking Water Administrators provides information on states with proposed or established standards. ${ }^{12}$

In addition to an absence of a drinking water regulation, there are no established clinical reference levels for PFAS. This provides uncertainty by providers looking to manage cases of mothers exposed to PFAS and their children. Since there is no consensus related to long-term medical management for those exposed to levels higher than background, reducing (and preventing) exposure is the critical intervention.

This can be accomplished by offering guidance to families about how to identify and reduce current sources of PFAS exposure, including drinking water and consumer products. When health concerns arise that might be associated with PFAS, it is important to conduct a complete history, including exposure assessment and physical exam relative to any symptom reported and manage the issue with established evidence-based guidelines (e.g., routine cholesterol screening; thyroid function tests if patient has symptoms consistent with thyroid dysfunction)..$^{13}$

Health providers should determine the need for monitoring based on risk factors such as age, source of drinking water, consumer products used, and occupational exposures. There is no known treatment once exposure occurs, so prevention of exposures is critical. In the absence of federal regulation, clinicians can provide pregnant women and families with resources and a number of strategies for reducing exposures including:

- Water filtration of contaminated water sources by using granulated activated carbon (GAC) and/or reverse osmosis (RO). ${ }^{14}$

- Dust control in the home (including wet mopping, wet dusting, and vacuuming) to reduce exposure from PFAScontaminated house dust.

- Avoid purchasing and using products with PFAS such as nonstick pans, waterproof garments, and stain-resistant carpets.

- Check local fish advisories in your community, as some states have fish consumption advisories for certain water bodies where PFOS has been detected in fish.

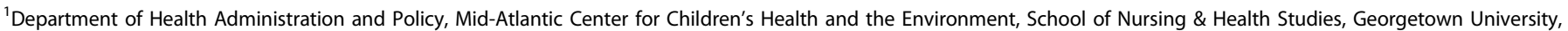
Washington, DC, USA

Correspondence: Laura Anderko (la266@georgetown.edu)

Received: 10 December 2019 Accepted: 13 January 2020

Published online: 14 March 2020 
The Centers for Disease Control and Prevention (CDC) and Agency for Toxic Substances and Disease Registry (ATSDR) measures PFAS as part of its national biomonitoring program. Current PFAS levels can be found in its 2018 report. ${ }^{15}$ Recently, CDC/ATSDR launched a nationwide multisite risk assessment to expand the science about the relationships between PFAS exposure near current or former military installations ${ }^{16}$ and is beginning to conduct a multisite study on the human health effects of exposures to PFAS through drinking water. ${ }^{17}$ The goal of the health assessment study is to learn more about the relationship between PFAS exposure and health outcomes among differing populations. Findings can be used to advance the science, and by health professionals to help address health issues in communities impacted by PFAS.

While research continues to emerge on health outcomes associated with PFAS exposure, the Pediatric Environmental Health Specialty Unit national network (PEHSU) is working closely with CDC/ATSDR to provide the most up-to-date information to guide clinicians' practice and protect the public's health. Dedicated to educating health professionals and the general public about reproductive and children's environmental health issues, the federally funded PEHSU national network offers fact sheets, webinars, and lectures to discuss emerging research and assist in translating findings to clinical practice and public health protections. ${ }^{18}$ Ultimately, healthy public policy that regulates PFAS is needed to reduce exposures and health impacts negatively impacting children.

\section{ACKNOWLEDGEMENTS}

This study is supported by the Agency for Toxic Substances and Disease Registry (ATSDR), cooperative agreement award number 1U61TS000238-05 (to the Region 3 Mid-Atlantic Center for Children's Health and the Environment). The contents of this manuscript are the responsibility of the authors and do not necessarily represent the official views of the Agency for Toxic Substances and Disease Registry (ATSDR). The U. S. Environmental Protection Agency (EPA) supports the Pediatric Environmental Health Specialty Units (PEHSU) by providing funds to ATSDR under Inter-Agency Agreement number DW-75-92301-05. Neither EPA nor ATSDR endorse the purchase of any commercial products or services mentioned in PEHSU publications.

\section{ADDITIONAL INFORMATION}

Competing interests: The author declares no competing interests.

Publisher's note Springer Nature remains neutral with regard to jurisdictional claims in published maps and institutional affiliations.

\section{REFERENCES}

1. Kingsley, S. L. et al. Variability and predictors of serum perfluoroalkyl substance concentrations during pregnancy and early childhood. Environ. Res. 165, 247-257 (2018).

2. Ye, X. et al. Per- and polyfluoroalkyl substances in sera from children 3 to 11 years of age participating in the National Health and Nutrition Examination Survey 2013-2014. Int. J. Hyg. Environ. Health 221, 9-16 (2017).

3. Blencowe, $\mathrm{H}$. et al. Preterm birth-associated neurodevelopmental impairment estimates at regional and global levels for 2010. Pediatr. Res. 74(suppl 1), 17-34 (2013).

4. Bach, C. C. et al. Perfluoroalkyl and polyfluoroalkyl substances and human fetal growth: a systematic review. Crit. Rev. Toxicol. 45, 53-67 (2015).

5. Manzano-Salgado, C. B. et al. Prenatal exposure to perfluoroalkyl substances and cardiometabolic risk in children from the Spanish INMA birth cohort study. Environ. Health Perspect. 125, 097018 (2017).

6. Barker, D. J. The developmental origins of adult disease. J. Am. Coll. Nutr. 23, 5885-5955 (2004).

7. Ratnasingham, A., Eiby, Y. A., Dekker Nitert, M., Donovan, T. \& Lingwood, B. E. Review: Is rapid fat accumulation in early life associated with adverse later health outcomes? Placenta 54, 125-130 (2017).

8. Environmental Protection Agency (EPA). Drinking Water Health Advisory for Perfluorooctanoic Acid (PFOA) 103 (Environmental Protection Agency (EPA), 2016).

9. Federal Register. Lifetime Health Advisories and Health Effects Support Documents for Perfluorooctanoic Acid and Perfluoro-octane Sulfonate. EPA-HQ-OW-2014-0138. 33250-33251 (2016).

10. New York State Department of Health. Drinking water quality council recommends nation's most protective maximum contaminant levels for three unregulated contaminants in drinking water. https://www.health.ny.gov/press/releases/ 2018/2018-12-18_drinking_water_quality_council_recommendations.htm (2018).

11. Minnesota Department of Health. Health department issues new guidance values for two perfluoroalkyl substances (PFAS). https://www.health.state.mn.us/news/ pressrel/2019/water040319.html (2019).

12. Association of State and Drinking Water Association. Per- and polyfluoroalkyl substances (PFAS) and State Drinking Water Program challenges. www.asdwa. org/wp-content/uploads/2019/04/ASDWA-PFAS-2-Pager-March-2019.pdf (2019).

13. Agency for Toxic Substances and Disease Registry (ATSDR). PFAS: an overview of the science and guidance for clinicians on per- and polyfluoroalkyl substances (PFAS). https://www.atsdr.cdc.gov/pfas/docs/ATSDR_PFAS_ClinicalGuidance_12202019.pdf (2019).

14. Anumol, T., Clarke, B. O., Merel, S. \& Snyder, S. A. Point-of-use devices for attenuation of trace organic compounds in water. J. Am. Water Works Assoc. 107, E474-E485 (2015).

15. Centers for Disease Control and Prevention (CDC). Fourth National Report on Human Exposure to Environmental Chemicals, Updated Tables (US Department of Health and Human Services, Atlanta, GA, 2018).

16. Centers for Disease Control and Prevention (CDC). PFAS exposure assessments. https://www.atsdr.cdc.gov/pfas/PFAS-Exposure-Assessments.html (2019).

17. Centers for Disease Control and Prevention (CDC). CDC/ATSDR PFAS related activities. https://www.atsdr.cdc.gov/pfas/related_activities.html\#Multi-Site-Health-Study (2019).

18. Centers for Disease Control and Prevention (CDC). Pediatric Environmental Health Specialty Units (PEHSU). https://www.pehsu.net (2019). 